\title{
ac2lus: Bringing SMT-solving and Abstract Interpretation Techniques to Real-Time Calculus through the Synchronous Language Lustre
}

\author{
Karine Altisen Matthieu Moy \\ Email: \{Karine.Altisen,Matthieu.Moy\}@imag.fr \\ Verimag (Grenoble INP), Grenoble, France
}

\begin{abstract}
We present an approach to connect the Real-Time Calculus (RTC) method to the synchronous data-flow language Lustre, and its associated tool-chain, allowing the use of techniques like SMT-solving and abstract interpretation which were not previously available for use with RTC. The approach is supported by a tool called ac2lus. It allows to model the system to be analyzed as general Lustre programs with inputs specified by arrival curves; the tool can compute output arrival curves or evaluate upper and lower bounds on any variable of the components, like buffer sizes. Compared to existing approaches to connect RTC to other formalisms, we believe that the use of Lustre, a real programming language, and the synchronous hypothesis make the task easier to write models, and we show that it allows a great flexibility of the tool itself, with many variants to fine-tune the performances.
\end{abstract}

Keywords-Real-Time Calculus, Lustre, Modular Performance Analysis, Observer, Formal methods

\section{INTRODUCTION}

Modern real-time embedded systems are increasingly complex and heterogeneous. Due to real-time requirements, the timing performances require accurate evaluation that help taking or validating decisions on the conception of a system as early as possible in the design process. Many modeling and analysis techniques have been developed among which we can distinguish two families. Computational approaches study fine-grain models of the system to represent its complete behavior. The validation of the system using such a model may involve simulation, testing and verification. Simulating precisely an embedded system gives very precise results, but only for one simulation, and one instance of a system. Formal methods such as model-checking, when possible, quickly face the state explosion problem if they are not associated to modular techniques. As opposed to this, analytical techniques, such as Real Time Calculus [1], use purely analytical models, based on mathematical equations that can be solved efficiently. These models can represent in a simple way the amount of events to be processed and how fast the processing occurs. Solving these equations is very fast and may provide, for example, the best and worst cases for performances. The main drawbacks of those techniques is that they imply rough abstraction of the system, leading to results that may lack accuracy. For example, Real-Time Calculus cannot handle the notion of state in the modeling of a system. Recent studies try to compositionally combine the approaches to take the best of both [2], [3], [4]. We present in this paper another way of combining RealTime Calculus with computational models, in the case of synchronous programs written in Lustre [5].

Real-Time Calculus (RTC): RTC [1] is a framework to model and analyze heterogeneous system in a compositional manner. It relies on the modeling of timing properties of event streams and available resources with curves called arrival curves and service curves. A component can be described with curves for its input stream and available resources and some other curves for the outputs. For already-modeled components, RTC gives exact bounds on the output stream of a component as a function of its input stream. This result can then be used as input for the next component.

An arrival curve is an abstraction to represent the set of event streams that can be input to (resp. output from) a component; it is expressed as a pair of curves $\left(\alpha^{l}, \alpha^{u}\right)$. For $k \geq 0, \alpha^{l}(k)$ and $\alpha^{u}(k)$ respectively provide, for any potential stream, the lower and upper bounds on the number of events that occur during any time interval of size $k$. Similarly, the processing capacity of a component is specified by a service curve $\left(\beta^{l}, \beta^{u}\right)$. The number of events that may be processed in any time interval of size $k$ is at least $\beta^{l}(k)$ and at most $\beta^{u}(k)$.

These curves do not allow to consider sequences of different abstract behaviors, since it is based on information valid for all intervals of a given length. This also implies that no notion of states is allowed in RTC components. When using such abstractions for components with several intrinsic modes, the obtained results are particularly coarse. For example, a component with an initial mode and then a stationary behavior cannot be described without unreasonable approximation.

Interfacing RTC with other formalisms: to overcome these drawbacks, a commonly used approach is to individually analyze some state-based or new component of an RTC framework with tools that support it and to re-inject the results into the RTC framework. This implies making the interfaces between those tools and 
the RTC analysis compatible, namely, each component inputs and outputs arrival curves.

This approach has already been used successfully to connect RTC to various other formalisms in the past: [6] proposed a connection to a simulation model that allows testing models or actual embedded systems. [7] presents a connection to event-count automata and [3] a connection to timed automata: they both represent the component and the input arrival curve as event-count (resp. timed) automata and then use a model checker to compute the output arrival curve. The connection with timed automata has been further improved to reduce the size of the models by focusing on a particular shape of curves [2] or by changing the granularity of events [4].

Overview of the approach: in this paper we propose to extend the RTC interfacing the Lustre synchronous data-flow programming language. When some component is well-suited for modeling and analysis with Lustre and its toolbox, we propose to individually analyze it and then to use the results in the RTC framework. Other components can still be analyzed with the usual RTC toolbox. We develop a method to analyze Lustre programs interfaced with RTC, with many options that can be tried to obtain some better results. A tool called ac2lus supports the framework.

An RTC component can be seen as an arrival curve transformer: taking some arrival curve as input, this specifies an output arrival curve for the component (see Figure 1 (a)). We now improve the expressiveness of the component by writing it in Lustre. This implies to make the interfaces between the Lustre component and the arrival curves compatible. Indeed, the new component, as Lustre is dataflow, computes on a given stream of events, whereas arrival curve describes a set of event streams in a very declarative way using relative time. To be able to come back and forth between the Lustre component and the arrival curves, we develop adapters and back adapters between them (see Figure 1 (b)).

We then run the analysis to compute the output arrival curve. It is done with an abstract interpretation tool (nbac [8]) and a model-checker (kind [9]) doing bounded model-checking and $k$-induction via SMT-solving ("Satisfiability Modulo Theory", i.e. SAT + a numeric solver).

We develop the tool ac2lus. It provides a library of predefined Lustre components (greedy processing components, fixed-priority scheduler, simple examples of power-managed components...) that the user can use to model a system. Some other components can easily be programmed. The user also provides the input arrival curves which ac2lus uses to generate the Lustre code for adapters and back-adapters. The tool implements some runners for the verification tools, which allows to compute the output arrival curves automatically.

The first goal of the approach it to extend the RTC framework by the local analysis of some Lustre component. It can also be used to evaluate some logical or quantitative property on the component. For example, given some arrival curve for a Lustre component, one can use the tool to compute the maximum buffer fill-level.

A similar approach was followed by [10]: they compute maximum delays of data-acquisition modules using Lustre models and abstract interpretation. The main differences with our work are that ac2lus allows modular analysis through the use of RTC arrival curves and enables the evaluation of any quantitative measure expressed in the Lustre model whereas [10] is bettersuited to compute maximum delay.

Contributions: we propose a new combination of the RTC framework with Lustre components which is fully automatized by the tool ac2lus. It increases the variety of validation techniques (abstract interpreters, SMT-solvers) available for analysis: if some system fails to be analyzed using some tools, one can change his strategy and try another one. With the same idea, the whole framework contains many variants and is easily configurable and extensible.

Comparing to the classical RTC analysis, those components are more expressive and allow to design statebased systems which is mandatory while studying e.g. power aware systems; in this case, the analysis via Lustre leads to much more precise evaluations than RTC.

Comparing to other state-based RTC interfacing frameworks, and in particular with [2] which is the most recent and the closest of our work :

- the shape of the curves they take into account is a bit less general (since we enable points between segments) and they strictly focus on discrete events whereas we can also handle fluid event models by changing to real the typing of event streams (with similar performance);

- we believe that the use of a programming language instead of any other formal models, makes the modeling of new components more intuitive and easier;

- the efficiency of the analysis and its precision are highly correlated, for both approaches. When the analysis succeed, the precision is the same since it is optimal. The performances bottleneck, in both cases, are due to the use of formal validation tools and to the size of the models. As usual, tools behave differently for various shapes of models and it is good having several strategies to try. For example, our approach scales nicely with the order of magnitude of numerical constants, while [2] scales better with respect to the timing constants.

Organization: the paper is organized as follows. Section II gives some details on the Lustre language and shows some RTC components written in Lustre; Section III details the adapters and back-adapters, explains the tool and the analysis; Section IV shows applications of the framework, illustrated on an example and Sec- 


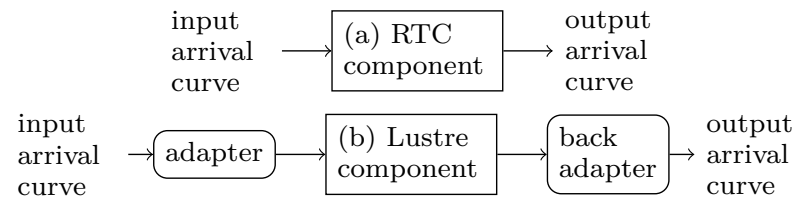

Figure 1. From RTC analysis (a) to other analysis (b)

tion $\mathrm{V}$ gives conclusions and future works.

\section{LUSTRE}

We use the synchronous data-flow language Lustre [5] to program the component to be analyzed. This is a simple but formally defined language which is dedicated to program embedded system or abstractions of it.

The motivation for choosing Lustre is multiple. First it is supported by a wide variety of validation tools such as model-checkers [11], [9], abstract interpreter [8], testers and simulators. By connecting RTC to the Lustre language, ac2lus opens the door to several other formal methods and tools that were not previously available within RTC. Second, the execution model of Lustre fits the RTC model rather well: both are dataflow-oriented. A Lustre program computes directly on event streams, hence, the constraints expressed by arrival curves correspond directly to properties of Lustre flows. This allows to program the interface (adapters) with arrival curve using the same language as the components; we use the technique of the so-called synchronous observers [12] to express the constraints of the arrival curve as a safety property of an event stream. Finally, an interesting property of Lustre is its simplicity of use. Lustre is not only a modeling language, but also an implementation language, that has already been successfully used to implement large systems. We believe that the use of an actual programming language makes the task of writing models for components easier than other formalisms.

Lustre programs manipulate infinite streams of values. When one writes $\mathrm{x}=\mathrm{y}+2$; it should be read as "at each clock tick, the value of $\mathrm{x}$ is equal to the value of $\mathrm{y}$ plus 2", so if the stream of values for y is $1,5,12,42, \ldots$, then the stream of values for $\mathrm{x}$ is $3,7,14,44, \ldots$ It is also possible to refer to past values of a variable, by using the operator pre which means "previous value". Since "previous value" is meaningless at the first instant, the operator pre has to be used together with the "initialization operator" $\rightarrow: \mathrm{x} \rightarrow \mathrm{y}$ means " $\mathrm{x}$ at the first instant, and y afterwards".

A Lustre function is called a node; it takes and computes streams of values as input and output parameters. Figure 2 shows a simple node which computes identity on a stream of integers.

Model of a Lustre Component: to model a component in Lustre, we naturally represent its input/output event streams with input/output Lustre flows. As Lustre

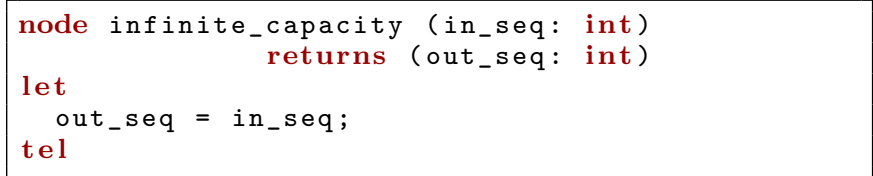

Figure 2. Simple Lustre Node

node gpc (in_seq: int; in_res: int)

returns (out_seq: int; out_res: int) var

backlog: int; work: int;

empty_queue: bool;

let

-- events to compute at the current

- instant (accumulated + new work)

work = in_seq $\rightarrow($ in_seq + pre $($ backlog $))$;

-- whether we'll empty the queue at the

-- current instant

empty_queue = (work <= in_res);

-- amount of work accumulated in the past

backlog = if (empty_queue) then 0

else work - out_seq:

- events produced

out_seq = if (empty_queue) then work else in_res;

-- resource remaining after running

tel

out_res = in_res - out_seq;

Figure 3. Greedy Processing Component in Lustre

programs computes a value "at each clock tick", time is obviously discrete. At each clock tick, a given amount of events arrives to (resp. is output by) the component. We do not consider individual events, but instead consider the number of events occurrences during a clock tick.

The node in Figure 2 can be seen as the Lustre model of a component with an infinite processing capacity: it receives events and immediately process them.

We consider the case where event count are integers: this model is usually called the discrete event model, as opposed to the fluid event model where the amount of data to process and resource can be continuous. Extension to the fluid model would be straightforward by replacing int Lustre flows with real.

A Common RTC Component in Lustre: to illustrate and validate the approach, we first model well-known RTC components in Lustre. A typical example of RTC component is the so-called Greedy Processing Component $(G P C)$. It models a process that enqueues the incoming events in a buffer and treats the events in a greedy fashion while being restricted by the available resource. It can be modeled in Lustre by the program in Figure 3, while in_res represents the maximum number of events that can be processed at this instant, and out_res the unused resource during this clock tick.

Other examples of Lustre components, that RTC cannot model because of states, will be given in Section IV.

\section{INTERFACING LUSTRE AND RTC}

In this section, we explain how to interface a Lustre component $C$ with RTC arrival curves. This means that 
we have to transform the constraints of the input arrival curve $\alpha_{i n}$ into explicit Lustre flows that complies with those constraints; those flows will then be input into $C$. On the other side, we have to express a set of explicit flows coming out of $C$ into constraints for the output arrival curve $\alpha_{\text {out }}$.

In fact, for the output part, we do not directly infer $\alpha_{\text {out }}$ from the outputs of the component. We build a candidate arrival curve and then check with a verification tool if it conforms to the output streams. The building for the candidate curve is done with a binary search procedure. This method allows to apply the same technique at the input and at the output; this choice is mainly due to the proof engines we use which are able to verify properties. A valuable extension to our work would be to try a tool such as aspic [13] which is able to infer numerical invariants on the program, from which we could deduce $\alpha_{\text {out }}$.

The technique we use is to characterize an arrival curve with an observer encoded by a Lustre node. Observers do not compute a curve, but they give a yes/no answer to the question "is the event stream compliant with the curve?".

The models of observers we propose are restricted to either finite specification of an arrival curve, namely a finite number of points specifies the curve (individual values $\alpha^{u}(1), \alpha^{u}(2), \ldots$ and $\left.\alpha^{l}(1), \alpha^{l}(2), \ldots\right)$, a piecewiseaffine function, which should be convex for lower-curve and concave for upper-curve (i.e. $\alpha^{u}(\delta)=\min \left\{a_{1}^{u} \delta+\right.$ $\left.b_{1}^{u}, a_{2}^{u} \delta+b_{2}^{u}, \ldots\right\}$ and $\left.\alpha^{l}(\delta)=\max \left\{a_{1}^{l} \delta-b_{1}^{l}, a_{2}^{l} \delta-b_{2}^{l}, \ldots\right\}\right)$, or a combination of both. We call the individual $\alpha^{u}(i)$ and $\alpha^{l}(i)$ values points, and the linear portions of curves $\alpha^{u}(\delta)=a_{i}^{u} \delta+b_{i}^{u} \alpha^{l}(\delta)=a_{i}^{l} \delta+b_{i}^{l}$ segments. Figure 4 shows an example with 3 points, two segments for the upper part, 3 points, one segment for the lower part.

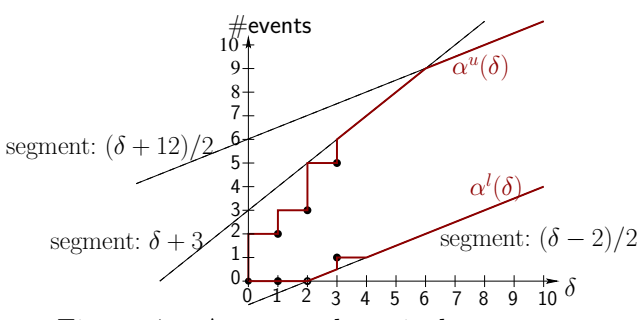

Figure 4. An example arrival curve

In the rest of the section, we first give a generic modeling of an arrival curve that is available for input and output adapter, called deterministic observers. Afterwards, we show how to use them to actually search for the best valid curve and describe the ac2lus tool which performs the overall computation. We finish with some variants for the node describing either the input adapter or the output adapter.

\section{A. Adapters using Deterministic Observers}

In the family of synchronous languages, where the communication between parallel components is the synchronous broadcast, observers [12] are a powerful and well-understood mechanism: an observer is a node that observes the inputs and the outputs of another node and computes some safety property. Here, we model the set of event streams that satisfy an arrival curve with a deterministic observer (see Figure 5 for the node). The observer inputs a stream of events called sequence and checks whether it satisfies or not the constraints of a given arrival curve (it outputs the Boolean stream ok). It exactly characterizes the arrival curve, by the property: an input stream sequence satisfies the curve iff ok remains true forever. The property to be expressed (and the code of the node) is divided into subproperties, one for checking compliance with the points of the curve and one per segment to be satisfied. The last line of the

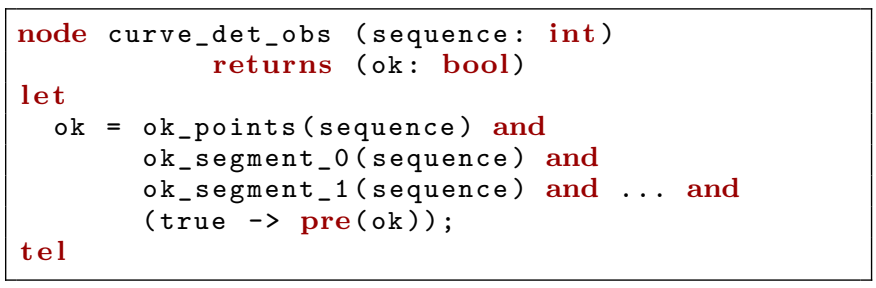

Figure 5. Deterministic Observer

node ensures the property of permanent failure: as soon as the arrival curve is falsified, ok remains false forever.

Deterministic Observer for Points: the part of the arrival curve specified by the points (let us say $N$ points) expresses $N$ constraints, one per time interval from size 1 to size $N$. The idea of the deterministic observer is to use one counter c $i$ per size $i$ of interval and to check its constraint. c $i$ represents the amount of events that occurred during the $i$ previous instants. At each new instant, it is updated by the previous value of $\mathrm{c}(i-1)$ (the number of events in the former window of size $(i-1))$ plus the amount of events that occurred at the instant (current value of sequence). The constraint to be verified is: for each $i$, c $i$ is between $\alpha^{l}(i)$ and $\alpha^{u}(i)$.

The node shown in Figure 6 is the deterministic observer for the points of the example curve of Figure 4. The additional variables inter $i$ are used at the beginning of the execution: for example, during the first two instants, the constraint on c3 has no sense since it should hold on interval of size 3 . inter $i$ expresses the fact that less than $i$ instants elapsed since the beginning of the execution. It can be computed with bounded integers or Boolean encoding (as shown in the code), leading to different analysis performances.

Deterministic Observer for Segments: the parts of the curves expressed by $\alpha^{u}(\delta)=\min \left\{a_{1}^{u} \delta+b_{1}^{u}, a_{2}^{u} \delta+\right.$ $\left.b_{2}^{u}, \ldots\right\}$ and $\alpha^{l}(\delta)=\max \left\{a_{1}^{l} \delta-b_{1}^{l}, a_{2}^{l} \delta-b_{2}^{l}, \ldots\right\}$ can 


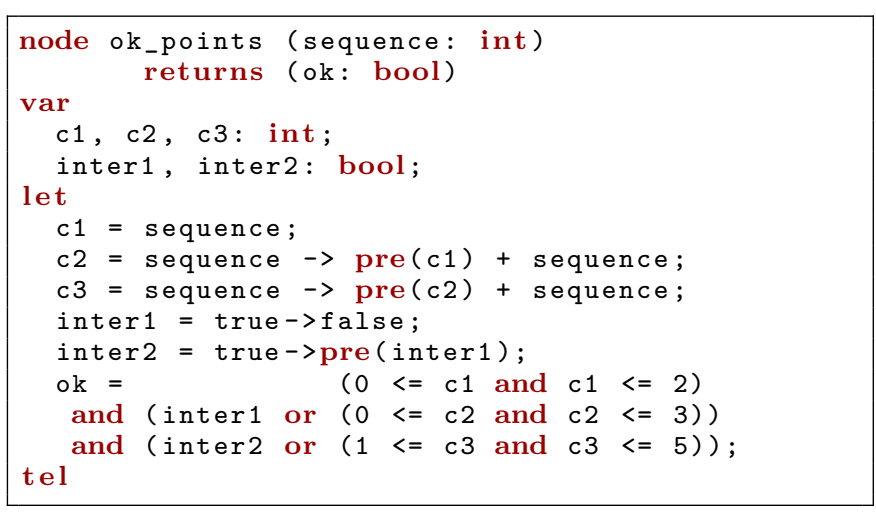

Figure 6. Deterministic Observer for Points

be expressed as a conjunction of observers for affine functions $a_{i} \delta+b_{i}$, where $a_{i}$ and $b_{i}$ are constant. We call each observer a segment observer. The codes for lower and upper segments are a bit different but both use the common principle of the leaky bucket. We explain it for the upper segment, and the reader can refer to [14] for the explanation of the lower curve.

The constraints from the arrival curve expresses the fact that the cumulated amount of events must stay below the segment. We model it as a bucket, whose content correspond to the number of events that can be emitted in burst at the current instant. It initially contains $b_{i}$ events. At each clock tick, the bucket is refilled by $a_{i}$ events, and the amount of events that occurred is poured off. After this computation, the bucket's content must still be within $\left[0, b_{i}\right]$ : if the new value is above $b_{i}$, the extra events are lost, and if it gets below 0 , it means the arrival curve is violated.

Figure 7 shows the generic code for the upper segment. The scale factor is a constant input which is used to keep the variables integer, while allowing $a_{i}$ to be rational.

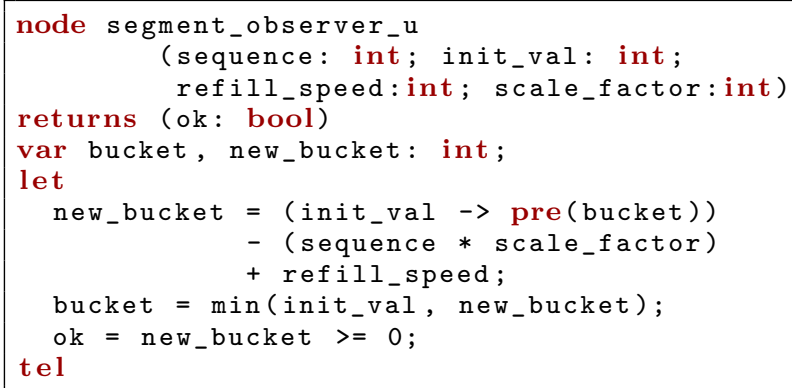

Figure 7. Deterministic Observer for an Upper Segment

The lower segment observer segment_observer_l is slightly different: the bucket is initially empty, and whenever the bucket's content crosses $b_{i}$, the property is violated. The lower segment does not give upper bound on the number of events, hence, emitting more events than the bucket's capacity is allowed, and results in an empty bucket.
The observer for the first upper segment of the curve in Figure 4 simply instanciates the generic observer: ok = segment_observer_u(seq, 3, 1, 1);

The leaky bucket idea comes from the ancestor of RTC, namely Network Calculus [15], and was already applied to the connection of RTC to computational models in [2], which directly inspired this part. The synchronous hypothesis greatly simplifies the implementation, which becomes just 3 simple Lustre equations including the property itself. Furthermore, the use of synchronous observers here makes the combination of segments easy: to describe a curve made of multiple segments (up and/or low), one can simply use an arbitrary Boolean combination of observers. Unlike [2], we do not have to introduce an additional scheduler automaton which does the combination by scheduling the "must emit" and "can emit" signals emitted by different automata.

\section{B. The analysis procedure}

This paragraph explains the procedure to compute one output arrival curve $\alpha_{\text {out }}$, given an input arrival curve $\alpha_{\text {in }}$ and a Lustre component $C$. Although, the framework can handle multiple input and output arrival curves, we explain first the procedure for one input and one output, in this paragraph, and then extend it to multiple inputs and outputs in the next paragraph III-C.

We first express the property that the triple $\left(\alpha_{\text {in }}, C, \alpha_{\text {out }}\right)$ is conformant. The building of a candidate for $\alpha_{\text {out }}$ is done using a binary-search procedure.

Checking input and output arrival curves (together): the goal here is to validate that given an input arrival curve $\alpha_{i n}$, a Lustre component $C$ and an output arrival curve $\alpha_{\text {out }}$, this triple is conformant, namely: every streams that may be output from $C$, when it executes with input streams that satisfies $\alpha_{i n}$, should satisfy $\alpha_{\text {out }}$. For both $\alpha_{i n}$ and $\alpha_{\text {out }}$, we instantiate deterministic observer nodes which respectively output the Boolean streams ok_in and ok_out and we combine them to obtain a global observer. This observer expresses the simple property: ok $=$ ok_in $\Rightarrow$ ok_out.

Proving that the property is invariant shows that the triple is conformant, if $\alpha_{i n}$ has a good shape. Indeed, Lustre observers in general models safety properties and the above observer expresses that at each instant $t$, ok_in $(t) \Rightarrow$ ok_out $(t)$. But, the observer for $\alpha_{i n}$ (resp. $\left.\alpha_{\text {out }}\right)$ characterizes the constraints expressed by the arrival curve with: $\forall t$, ok_in $(t)$ (resp. $\forall t$, ok_out $(t))$. We thus need to express: $\left(\forall t, \mathrm{ok}_{-}\right.$in $(t) \Rightarrow(\forall t$, ok_out $(t))$ which is not equivalent to the former formula in general. However, the two formulations become equivalent if the following three conditions are verified [12]: Permanent failure (last line of Fig. 5), determinism of the precondition, and causality [16] (see [14] for details). 
Building the output arrival curve: the tools we are using for verification of the global model was designed to prove properties, not to discover invariants, so a little additional work has to be performed to compute the output analytic description based on the input ones. This step is based on a binary search, with an algorithm which tries different values until it finds the best ones which are provably correct.

Let us explain the procedure for building the points of the curve on an example. To compute $\alpha^{u}(4)$, we start with the hypothesis $\alpha^{u}(4)=0$, generate the observer for this particular curve (assuming $\alpha^{l}=0$ and $\alpha^{u}(t)=+\infty$ for $t \neq 4)$. If the curve is incorrect, we try with $\alpha^{u}(4)=$ 1 , and then 3,7 , (after trying value $n$, we try $2 n+1$ ) ... until we find one acceptable value. At that point, we have one provably correct value, and the previously tried one (or just 0) which is not, and we can proceed with a binary search between these two values. This implies launching the proof engine $\log (n)$ times for each point (where $n$ is the value to be computed), but this has not been a problem in practice.

The procedure is quite the same for building the segments of the curve. For each segment $a_{i} x+b_{i}$, the two values $a_{i}$ and $b_{i}$ has to be evaluated. We first fix $b_{i}$ to an arbitrarily big value, and perform a binary search on $a_{i}$, and then fix $a_{i}$ to the value just computed to find $b_{i}$. More details, and the description of some optimizations can be found in [14]

\section{The ac2lus Tool}

The approach is implemented in the toolbox ac2lus. We present here the structure of the tool, some more implementation details can be found in [14]. From the user point of view, one provides a component $C$ to analyze as a Lustre node, having any number of inputs $I_{i}$ and output $O_{j}$. For each input, one must provide an arrival curve $\alpha_{i}$. The tool computes the curves $\left(\alpha_{\text {out }}\right)_{j}$ for each output. The execution is done as follows.

For each $I_{i}$, the tool generates the Lustre code for the adapters (as described in section III-A, or using one of the variants below). Then, it generates the main Lustre node, which instantiates the input adapters, the component $C$, and the output adapters. This nodes defines the property to verify, which is of the form $\mathrm{ok}=\left(\bigwedge_{i} \mathrm{ok}_{-} \mathrm{in}_{i}\right) \Rightarrow\left(\bigwedge_{j}\right.$ ok_out $\left._{j}\right)$.

We then start the binary search. We compute each output independently. To compute the curve for $O_{j}$, we first generate stubs for each $O_{k}, k \neq j$ for which ok_out $_{j}=$ true, and apply the binary search algorithm of section III-B for $O_{j}$ to compute each of its points, and optionally a segment to describe the long-term rate. The search consists in trying values, and asking proof engines whether the value is a correct bound or not.
We delegate the verification of the model to different tools. The ability to deal with numerical variables is a must-have, hence plain model-checking (enumerative or symbolic with BDDs) is not an option. ac2lus can currently use the abstract interpretation tool nbac [8], and the kind [9] verifier which is based on $k$-induction and uses SMT-solving. From the user point of view, both accept Lustre programs as input, and can prove properties of the form "OK is always true". nbac works on an abstract domain, and cannot find counter examples, hence its output is either "true" or "don't know". The SMT solver used internally by kind actually provides a set of values when given a satisfiable problem, hence kind's output is either "true" or "false with counter example", or can sometimes reach a timeout.

By default, ac2lus launches kind on the generated code. If kind proves the property or finds a counterexample, we move on to the next value to try. If kind reaches a timeout then nbac is tried. Optionally, on failure of nbac, we try kind -loop which sometimes give better results.

\section{Variants for Adapters}

The adapter presented above has the advantage of being applicable both for inputs and outputs. However, this is not the only option. Some variants produce Lustre programs with different number of variables, some nondeterminism, and yield different results in the proofs. We give here the overall idea for each variant. The implementation details can be found in [14].

Generator: one commonly used approach to implement an adapter for the input of a component is to write a generator (as done in [2], [4], [7]), which instead of telling whether an arbitrary event stream is correct, generates a stream which is correct by construction (using non-determinism to be able to generate all possible streams). When using a generator (if one input and one output), the global property to prove is no longer "ok $=$ ok_in $=>$ ok_out", but simply "ok = ok_out".

Non-Deterministic Observer: while using observers, another variant is to allow non-determinism in the observer. In Lustre, non-determinism will be modeled with additional inputs called oracles. With deterministic observer, the property is false iff the observer outputs "OK = false" at some point in time. With a non-deterministic observer, the property is false iff there exist an oracle for which the observer outputs "OK = false" at some point in time. Such observer are obviously not convenient for testing, since showing a trace incorrect requires choosing an oracle, but are equivalent to deterministic observers for formal verification when computing the output curve (but as said above, they cannot be applied to the input).

At time $t$, the deterministic observer checks the number of events for all windows $[t-\delta, t]$. Instead, the non- 
deterministic observer can chose non-deterministically a time $t_{0}$ where it starts checking, and then at time $t>t_{0}$, checks only the window of time $\left[t_{0}, t\right]$. For any incorrect stream, there exists an oracle $t_{0}$ for which the observer emits "OK $=$ false". The advantage of this method is that it requires only one numerical variable to count the number of variables in the interval $\left[t_{0}, t\right]$.

Other Variants: in some cases, the Lustre code generated from arrival curves can be written differently to get better results in the proof engine. The variants we implemented are based on a trade-off between numerical and Boolean variables. The details are given in [4].

\section{Applications of the Framework}

This section shows applications of the framework and illustrates them on a concrete example. These applications are: Modular analysis analyzes the components of a complex system one by one, by using the computed output of the first component as the input for the analysis of the next one; Global analysis analyzes several components together to get better precision, but sacrificing performance; Invariant discovery by proving a property: in addition to computing arrival curves for the output of a system, one can directly prove properties on a component. With a simple binary search algorithm, one can find the best provable bounds for any variable of the Lustre program (for example, the buffer fill-level).

We show that ac2lus is able to give results for these 3 applications, in cases where RTC would not be applicable, or too approximate. When the proof engine is able to conclude, the results provided by ac2lus are optimal.

The experiments were made on a dual-core, Pentium D CPU running at $3.40 \mathrm{GHz}$, with $2 \mathrm{~GB}$ of RAM.

\section{A. A Simple power-aware component}

Our original motivation for this work was to be able to model power-managed components, which cannot be modeled and analyzed precisely in pure RTC, because their behavior is state-based.

Figure 8 gives an example of a simple power-managed component. It is not meant to be realistic, but illustrate a state-based behavior: the component starts in sleep mode, and wakes up only when its input buffer fill-level (backlog) reaches a certain threshold. When it starts processing, it does so according to its input resource until the buffer is emptied.

The state of the system is modeled in the equation for serving, which means: initially, the component is asleep, then, if it used to be serving, it remains so until the backlog is 0 , and otherwise, it goes in service state when the amount of work to do is greater than threshold. The other equations are a straightforward adaptation of the GPC component presented in Figure 3.

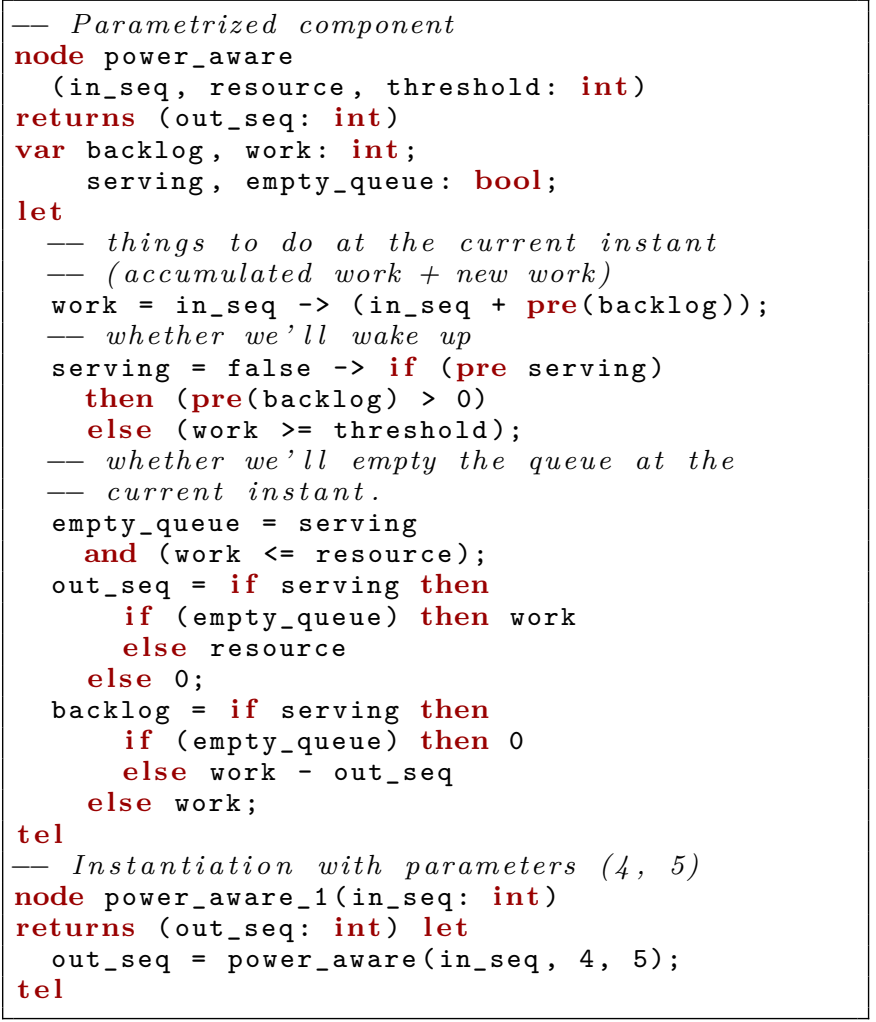

Figure 8. Lustre model for a simple power-aware component

The node power_aware_1 is basically an instantiation of the previous node, setting the threshold and resource to constant values.

Computing an Output Arrival Curve: we perform the analysis on the input curve: $\alpha^{u}(\delta)=\min \{9 \delta, \delta+$ $15\}$ and $\alpha^{l}(\delta)=\delta$. The analysis lasts 46 seconds, and launches kind 76 times. It gives the following result:

$\alpha_{\text {out }}^{u}=0,4,8,12,16,20,24,26,27,28,29$

$\alpha_{\text {out }}^{l}=0,0,0,0,0,2,6,6,6,6,6$

Here comes an explanation of the result on $\alpha_{\text {out }}^{u}$ : we can see a first linear fragment up to $\alpha_{\text {out }}^{u}(6)=24$, which corresponds to case where the component empties its buffer, at the speed of 4 events per time-unit. Starting from $\alpha_{\text {out }}^{u}(7)$, the rate decreases because the speed of the component is greater than the rate of arrival. Hence, the backlog is bounded, and the worst-case corresponds to the bursts of the input at the instant when the buffer crosses the threshold.

The result computed by the tool is optimal when kind answers (i.e. no time-out), namely, the obtained value is the tighter one. To explain this, let us consider a precise example: when trying to prove $\alpha_{\text {out }}^{u}(10) \stackrel{?}{=}$ 23 , kind concluded with a counter-example, which exhibits strictly more than 23 events in 10 time units. This counter-example corresponds to the input sequence $1,1,1,1,2,1,1,5,1,5,1,7,1,1$. Hence, 23 is provably not a valid bound, while $\alpha_{\text {out }}^{u}(10)=24$ is valid and thus the 
optimal value. In the complete example, kind concluded (with either true or false property) each time it was called, so this optimality result applies to each point of $\alpha_{\text {out }}^{u}$ and $\alpha_{\text {out }}^{l}$, which are therefore optimal.

Notice that examining the counter-examples produced by kind is of great help! It allows the user to better understand the results and to find non trivial cornercases leading e.g. to bursts or low number of emitted events. For example, on $\alpha_{\text {out }}^{l}$, the tool shows that it's not possible to remain in sleeping mode for more than 4 time units. The point $\alpha_{\text {out }}^{l}(5)=2$ corresponds to an execution where the component emits 2 events to finish emptying its buffer, and then emits nothing for some 4 time units. kind's counter-example for $\alpha_{\text {out }}^{l}(5) \neq 3$ shows that this lower bound is obtained for in_seq $=$ $2,3,1,1,1,1,1$, which leads to out_seq $=0,4,2,0,0,0,0$. Even on a simple system like this example, the above counter-example and the proof that $\alpha_{\text {out }}^{l}(5)=2$ could hardly be found manually.

Comparing with $R T C$, it would produce very coarse results on the same example. Indeed, it would not be able to handle the two modes of operation and thus could only consider the best-case and worst-case for the availability of resources: the worst-case is the sleeping mode, which does not compute at all, so we would get $\alpha_{\text {out }}^{l}(\delta)=0, \forall \delta$; in the other side, RTC service curves would not forbid the scenario where the component remains in sleeping mode, accumulating events in its buffer, for an arbitrarily long time, and therefore emit 4 events per seconds for an arbitrarily long time afterwards. Hence, the best we could get is $\alpha_{\text {out }}^{u}(\delta)=4 \delta$.

Quantitative properties to compute bounds on variables of the system: in addition to being able to compute output arrival curves, we can also prove properties on the component itself, and find bounds on any of the variable of the system. For example, to compute a bound on the buffer, we add backlog as an output of the module, and replace the output observer in the main node with the equation obs_ok_out_seq $=$ backlog $<=N$; By trying different values of $N$, we can find which is the best bound for backlog. On this example, a quick binary search yields $N=13$ for which the property is proved, and $N$ $=12$ gives the counter example in_seq $=8,9$. Hence, 13 is the best possible bound on the backlog.

The proof do not need to previously compute the output arrival curve and is very quik since it took 0,4 seconds. Notice also that RTC would not be able to get any bound on the backlog of this system.

To sum up on the example, we got the optimal values for $\alpha_{\text {out }}^{u}(\delta)$ and $\alpha_{\text {out }}^{l}(\delta)$ up to $\delta=10$, a bound for the size of the buffer, and counter-examples showing that any value more precise than the ones we computed would be incorrect, in less than a minute, in a case where $R T C$ does not apply.
Comparison of different versions of the adapters: this above experiment was performed with the default settings of ac2lus. Using any other leads to slower performance and/or computation of suboptimal curves, because the proof engine fails to conclude in some cases, as shown in the table below (see [14] for more details).

When using deterministic observers, kind appears to be strictly better than nbac: faster, and able to prove more properties. Conversely, when using nondeterministic observers, kind does not prove any of them, and nbac is the only tool giving a result.

\begin{tabular}{l|r|r} 
Default (deterministic observer) & $46 "$ & optimal \\
\hline Non-deterministic observer & 8 min5" & suboptimal \\
\hline Generator & 1 min26" & suboptimal \\
\hline $\begin{array}{l}\text { Non-deterministic observer and } \\
\text { generator }\end{array}$ & 6 min11" & suboptimal \\
\hline $\begin{array}{l}\text { Boolean encoding of segments } \\
\text { disabled }\end{array}$ & $2 \min 18 "$ & optimal \\
\end{tabular}

\section{B. Composing power-aware components}

Approach for Analyzing Complex Systems: the way to tackle complexity in RTC is to split a system into multiple small components, and reduce the analysis of the system to a set of local, simple analysis. ac2lus inherits from this possibility: a component is modeled with a Lustre node, having inputs and outputs, and the components can easily be plugged together.

This can obviously be used to model a system with several physical components, and applies particularly well when the architecture is well pipelined. Actually, this can also be used to model simple scheduling strategies: the Lustre flows can model event streams, but also resource units streams (denoted by $\beta$ ). Input flows represent the amount of resource available during a clock tick $(\beta)$, and the output flows give the amount of resource remaining after the modeled task has ran $\left(\beta_{\text {out }}\right)$. A fixed-priority scheduling between $N$ tasks can therefore easily be represented by connecting the output $\beta_{\text {out }}^{n}$ to the input $\beta^{n+1}$ of the next one (component $n$ having more priority than component $n+1)$. An example of a fixed-priority scheduler, re-using the GPC component defined in Figure 3 page 3, with two tasks is written as:

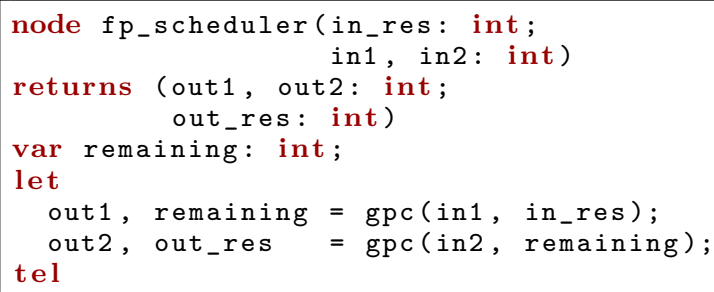

The connection from a component to another can be done in two ways. Either we compute the arrival curves for the output of the first component, and we 
apply the usual modular analysis for RTC (using a fixpoint computation for circular dependencies [17]), or we actually connect the Lustre nodes to do a global computation.

An Example of Two-Components System: we continue the experiment with a second load-dependent component: if its backlog is greater than 4, the component computes at the rate of 10 events per time unit. Otherwise, it computes only 1 event per time unit. The source code for this module is given in [14]. The node load_depend_gpc is defined following the principle of the GPC, but the incoming resource in_res is replaced with if (work $>=$ threshold) then res_fast else res_slow; in the equations.

We now consider a system composed of the two components. Assembling the components is done in a few lines of Lustre code:

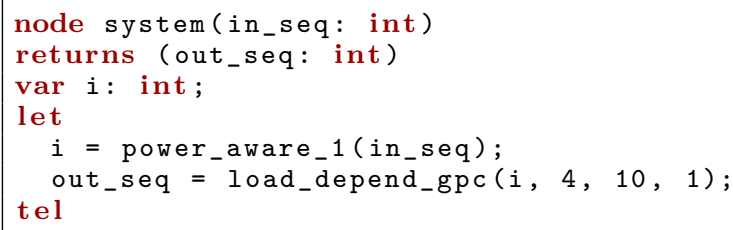

Running ac2lus on this system, with the same arrival curve as in the previous section for the input, we get the output (in $10 \mathrm{~min} 44$ "):

$$
\alpha_{\text {out }}^{u}=0,5,9,13,17,21,24,25,27,28,30
$$$$
\alpha_{\text {out }}^{l}=0,0,0,0,0,0,0,0,0,0,0
$$

In this case, the curves are approximate: we run the proof engine with a timeout of 10 seconds, and the results show only the provable points of the curves, within this timeout. In this experiment, the timeout has been reached when trying to prove the point $\alpha_{\text {out }}^{u}(9) \stackrel{?}{=} 27$ (kind proves $\alpha_{\text {out }}^{u}(9)=28$ it if we increase the timeout to 20). The points $\alpha_{\text {out }}^{l}(\delta)=0$ are provably the best points for $\delta \leq 4$, but the next points are approximate (and indeed, disappointing since the tool could not prove anything here).

Another option is to run the analysis in a modular way: first, analyze power_aware_1, get the output arrival curve, and use this arrival curve as input for the local analysis of load_depend_gpc. This approach gives different results:

$$
\begin{aligned}
& \alpha_{\text {out }}^{u}=0, \quad 6,10,14,18,22,26,28,80 \\
& \alpha_{\text {out }}^{l}=0,0,0,0,0,1,4, \quad 5,0
\end{aligned}
$$

The results are exact bounds given the input arrival curve up to $\delta=7$, and are approximate afterwards.

Performance-wise, the first points of the curves are computed much faster when using the modular method: computing the first 5 points takes 8 seconds modularly, and 34 seconds when done with the global approach. The complexity grows linearly with the number of components of the system.

The approach is flexible and allows multiple ways to analyze the same system. Obviously, since each analysis give a valid bound, the $\min / \max$ of all analysis is also valid and may give better bounds. For the example, combining both results, and applying subadditive closure, we get: $\alpha_{\text {out }}^{u}=0,5,9,13,17,21,24,25,27,28,30$ $\alpha_{\text {out }}^{l}=0,0,0,0,0,1,4,5, \quad 5,5,5$

\section{Evaluation and Comparison}

Apart from obtaining output arrival curves, the above experiments show that: the tool provides counterexamples which enable subtle understanding of the system; quantitative or logical properties can easily be expressed and directly analyzed with the tool; many strategies of the tool can be tried to obtain the tightest results.

Compared to [2], the closest approach to our work, component can be easily written and extended. For example, the fixed-priority scheduler is just 2 lines of Lustre, while the model with Timed Automata is far less trivial. Both tools use exact model-checking (we use abstract interpretation through nbac as a fall-back when kind fails). When the analysis succeeds, they both give the same result. But on both sides, the proof may be too long, and may have to be stopped with a timeout, leading to imprecision. The bottleneck is therefore the performance.

To the best of our knowledge, there exist no comprehensive benchmark for the problem we are solving, hence, we can compare only with a few examples, usually chosen to perform well with the tool they benchmark.

We tried the case study of [2] on ac2lus, but it showed bad results: we are able to compute a few points of the curves, but not enough to get relevant results, and the long-term rate ac2lus computes is over-approximated by a factor of 2 (the proof fails with a timeout for the actual long-term rate). Our interpretation of these bad results is that our model uses large timing constants (we wrote the model with a timing granularity of $1 \mathrm{~ms}$ ), and the number of variables we use is proportional to the order of magnitude of numerical constants. Uppaal, used by [2], uses an efficient symbolic encoding of time (based on zones), and doesn't suffer from this.

On the other hand, our experiments with timed automata and RTC [4] showed that the performance of the analysis was proportional to the order of magnitude of counters (since counters are managed enumeratively by Uppaal). ac2lus and the underlying model-checkers are far less sensitive to this. For example, we tried multiplying all the constants of the model (and arrival curves) of the system of section IV-B by 20 and rerun the analysis, which took $44 \mathrm{~min}$ instead of $6 \mathrm{~min} 11$ (dividing the result by 20 yields the same result as the initial one). The performance degradation is here less than linear with respect to the size of counters (this 
is partly due to the number of iterations of the binary search). With an enumerative algorithm, we would pay a factor 20 in four places: the generator, each of the two components, and the observer.

\section{Conclusion}

We presented ac2lus, a new combination of the RTC framework with the Lustre language. It allows to model the system to be analyzed (or parts of it) as Lustre components with inputs specified with arrival curves; the tool can compute output arrival curves using modular analysis or global analysis and it can also evaluate some quantitative property on any variable of the components. As other RTC interfacing techniques [2], [7], [3], this results in a considerable increase of the expressiveness of the framework, essential to model power-managed components, while keeping the modular aspect of RTC. Nevertheless, compared to them, ac2lus complements the state of the art by allowing a wider variety of modern methods to be used, namely Abstract Interpretation and SMT-solving. As a side effet, we believe that the use of a synchronous programming language makes easy for the user to write models, and allows a lot of variants of the adapters to fine-tune the performances.

In the future, we plan to try other verification tools as alternatives to kind and nbac. The tool aspic [13] sounds promising. It adds acceleration techniques to abstract interpretation, which should apply to the Lustre code we generate for adapters. Also, aspic does not only prove properties, but it is also able to discover invariants. This could allow us to get rid of the binary search, and launch the tool only once to compute a value.

An interesting application of ac2lus would be to consider the counter-examples provided by the proof engines for the last points which fails during the binary search. These counter-example exhibit an execution for which the computed bounds $\left(\alpha^{u}, \alpha^{l}\right)$ are actually reached. These executions can be interesting for diagnosis, and could also be used to generate test-cases for the actual system, since they exhibit corner-case behaviors, hardly reproducible randomly or manually.

Our long term goal is to be able to actually handle energy: handling state-based behavior in RTC allows proving timing properties on energy-aware systems, but still gives no information on energy consumption.

Finally, we are looking for alternatives to RTC's arrival curves to model abstraction of event streams. After introducing state-based behaviors in the components, we believe the next, logical step is to introduce the same in the interfaces between components.

\section{REFERENCES}

[1] L. Thiele, S. Chakraborty, and M. Naedele, "Real-time calculus for scheduling hard real-time systems," in $I S$ $C A S, 2000$.
[2] K. Lampka, S. Perathoner, and L. Thiele, "Analytic realtime analysis and timed automata: A hybrid method for analyzing embedded real-time systems," in EMSOFT, 2009.

[3] "Cats tool," 2007, http://www.timestool.com/cats.

[4] K. Altisen, Y. Liu, and M. Moy, "Performance evaluation of components using a granularity-based interface between real-time calculus and timed automata," in $Q A P L, 2010$.

[5] J.-L. Bergerand, P. Caspi, N. Halbwachs, D. Pilaud, and E. Pilaud, "Outline of a real time data-flow language," in $R T S S, 1985$.

[6] S. Künzli and L. Thiele, "Generating event traces based on arrival curves," in $M M B, 2006$.

[7] L. T. Phan, S. Chakraborty, P. Thiagarajan, and L. Thiele, "Composing functional and state-based performance models for analyzing heterogeneous real-time systems," in RTSS, 2007.

[8] B. Jeannet, "Dynamic partitioning in linear relation analysis. application to the verification of reactive systems," Formal Methods in System Design, 2003.

[9] G. Hagen and C. Tinelli, "Scaling up the formal verification of Lustre programs with SMT-based techniques," in FMCAD, 2008.

[10] L. Morel, J.-P. Babau, and B. Ben-Hedia, "Formal modelling framework of data acquisition modules using a synchronous approach for timing analysis," in WRTP/RTS, 2009.

[11] N. Halbwachs, F. Lagnier, and C. Ratel, "Programming and verifying critical systems by means of the synchronous data-flow programming language LUSTRE," Transactions on Software Engineering, 1992.

[12] N. Halbwachs, F. Lagnier, and P. Raymond, "Synchronous observers and the verification of reactive systems," in AMAST, 1993.

[13] L. Gonnord and N. Halbwachs, "Combining widening and acceleration in linear relation analysis," Lecture Notes in Computer Science, 2006.

[14] M. Moy and K. Altisen, "ac2lus: Bringing SMT-solving and abstract interpretation techniques to real-time calculus through the synchronous language lustre," Verimag, Tech. Rep. TR-2010-2, 2010.

[15] J.-Y. Le Boudec and P. Thiran, Network Calculus. Springer Verlag, 2001.

[16] K. Altisen and M. Moy, "Arrival curves for real-time calculus: the causality problem and its solutions," in TACAS, March 2010.

[17] B. Jonsson, S. Perathoner, L. Thiele, and W. Yi, "Cyclic dependencies in modular performance analysis," in EMSOFT, 2008. 